FLUID MAPPERS AS VISUAL ANALOGS FOR POTENTIAL FIELDS

\author{
By A. D. Moore \\ Department of Electrical Engineering, University of Michigan, Ann Arbor, Mich.
}

(1) Introduction

In a fluid mapper, streamline fluid flow is made to occur in a thin flow space between an upper and a lower member. The upper member is a flat piece of plate glass, called the plate. The lower member may be a slab of suitable material, usually plaster, or the surface of a "sandbed," or a combination of the two. The photographs reproduced herewith represent all three types. The fluid typically used is water. Normally, the two members are operated totally immersed in a tray of the operating fluid.

When a given potential field situation is to be portrayed, the lower member of the fluid mapper is built to scale, with suitable boundaries, open or closed; islands, if any; one or more sources or sinks; and so on. Each source or sink is connected by a rubber tube to a tank, so that raising or lowering a tank will induce flow in the flow space. When the operation is conducted so that the flow is not affected by inertia, the flow pattern set up can quite accurately duplicate either the equipotential lines, or else the flux lines, of the potential field under consideration.

Visible flow lines are produced, typically, by the use of potassium permanganate crystals. If the crystals are sprinkled onto the lower member prior to placing the plate, their dye lines move along the floor of the flow and form a floor pattern, as in FIGURES 1,4 , and 5 . If attached to the plate, a sharper set of lines is often obtained: the dye lines form a ceiling pattern at first, then sink slightly to be carried along at the higher velocities of lower levels in the flow space. Ceiling patterns are seen in FIGURes $2,3,6$, and 7 .

The writer invented the sandbed type of fluid flow analog in 1943, and proved it experimentally. That was the beginning of the modern work with the fluid flow analog. In 1948, the writer began the development of plaster techniques, and the devising of numerous forms of what the writer has called fluid mappers followed in rapid succession. Five papers (see references) have been published. Since these papers present many of the techniques and show many of the things that can be done with fluid mappers, the present paper will not attempt to include the details already published.

\title{
(2) The Analog
}

Going across the thin flow space vertically, the velocity distribution is parabolic. This particular phenomenon has no counterpart in any of the potential fields being simulated. In this respect, therefore, the fluid flow situation is not an analog for the potential field situation. But if visible dyeline patterns are formed at the floor and the ceiling of the flow, and at levels in between, it is found that all these patterns coincide when viewed from directly overhead. Professor Hele-Shaw ${ }^{1}{ }^{2}$ in England, through his beautiful pioneering work, showed experimentally and analytically that the flow patterns 


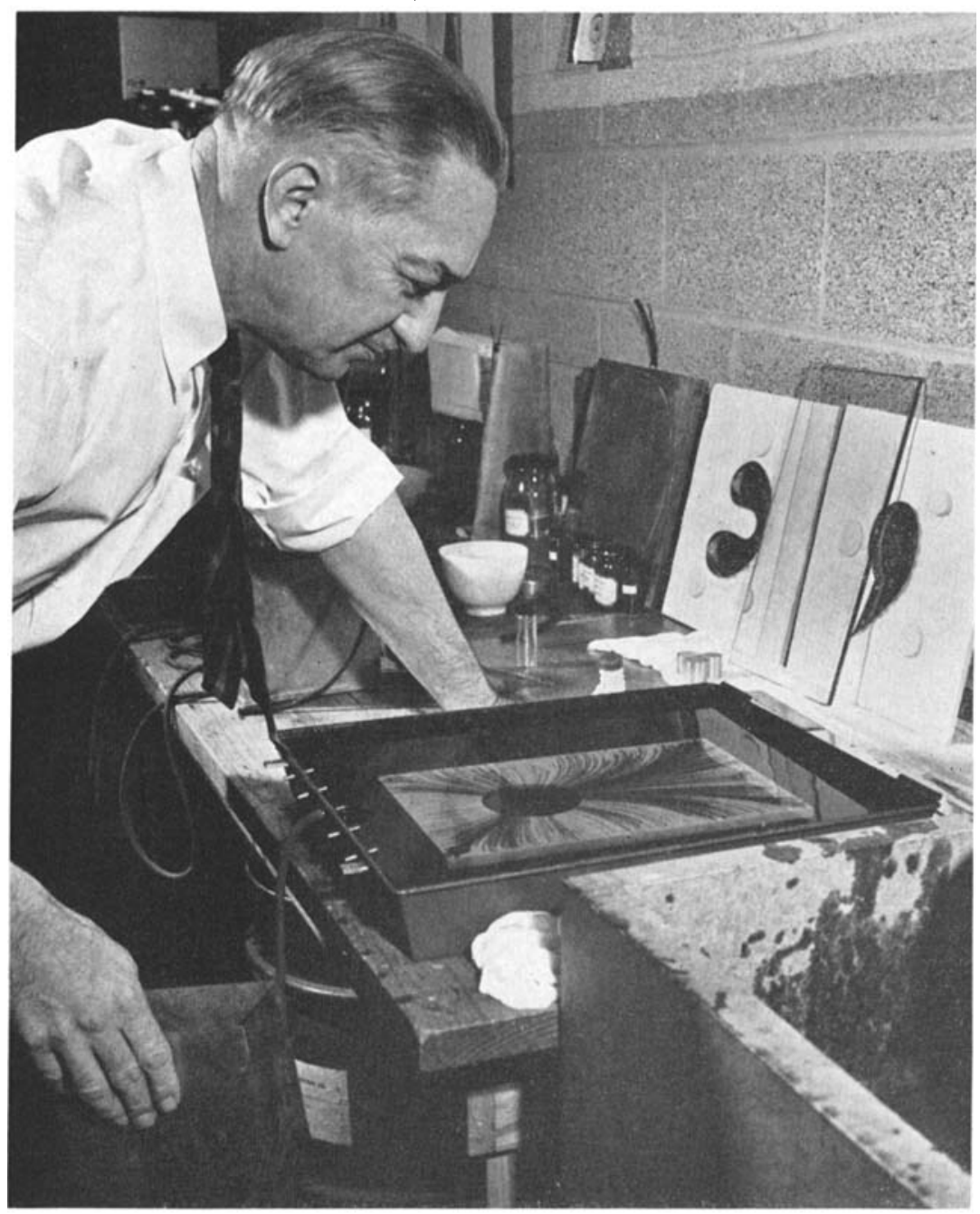

FrGURe 1. Fluid mapper in operation. The tank has been lowered to induce flow. The round well is acting as a sink.

thus set up do simulate the lines of the corresponding potential field. His work was done a half-century ago. His techniques, though admirable for their accuracy, were costly and inflexible, which may account for the fact that his work was not developed.

\section{(3) Slabs}

A slab, as shown in FIGURE 2, in theory, may be made of any suitable material. For demonstration purposes, however, and for many actual applications, 


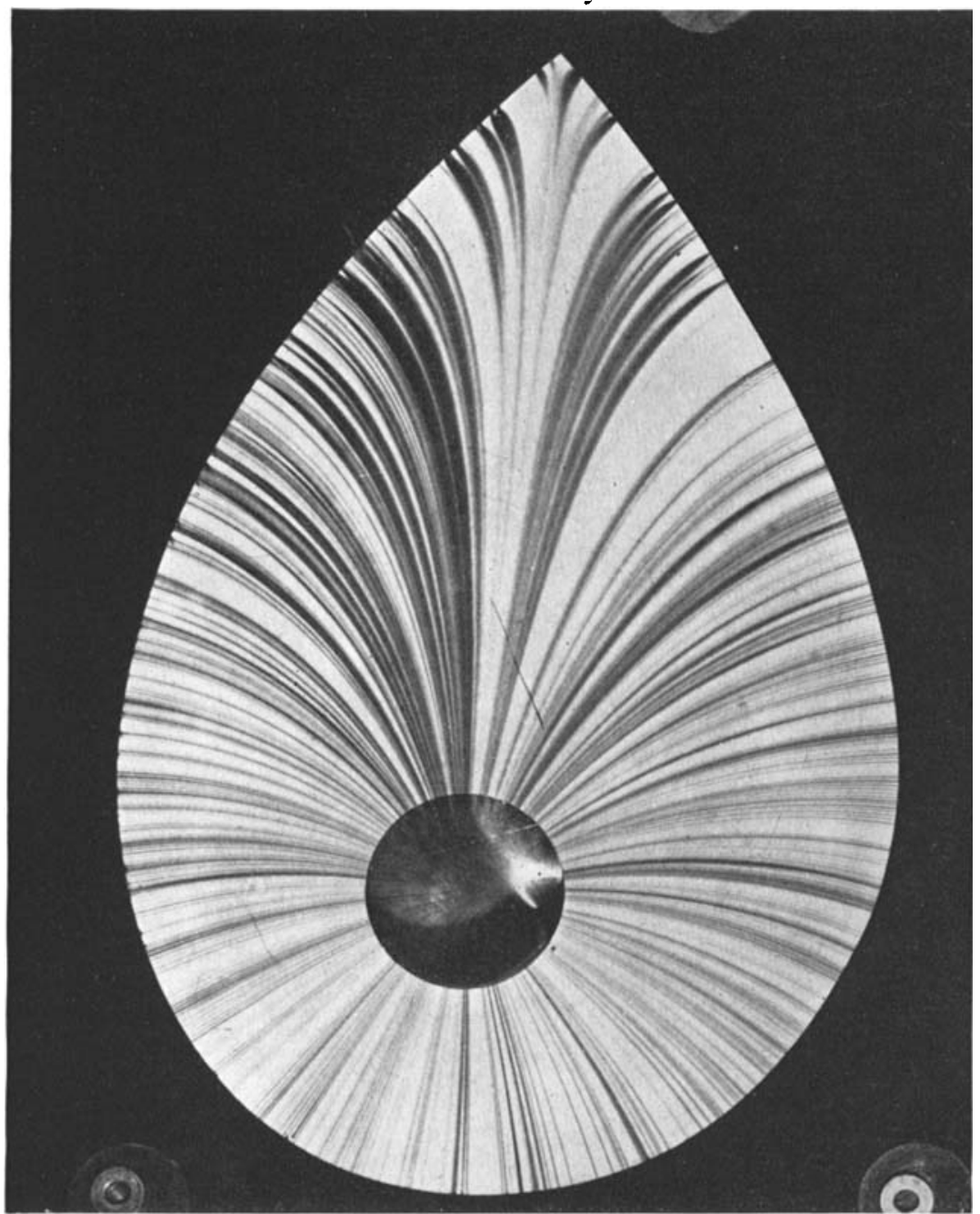

Figure 2. Fluid mapper with ceiling pattern. Crystals (invisible here) are attached to plate, just outside of outer boundary. The round well is acting as a sink.

plaster is by long odds the material of choice. By using techniques worked out by the writer, plaster slabs can be cast on plate glass to get a flat surface; plaster feet can be added; and plaster techniques can be used for finishing well bottoms and attaching the tubes to the wells at the same time. Thus, plaster is a cheap material of great adaptability.

Patching plaster can be, and has been used. Plaster of Paris may also be used, but the best plaster, as to setting time, strength, density, and accuracy, is a product that dentists call dental stone. Being mainly calcium sulfate, it is admittedly slightly soluble in water. For brief operations with water as the 


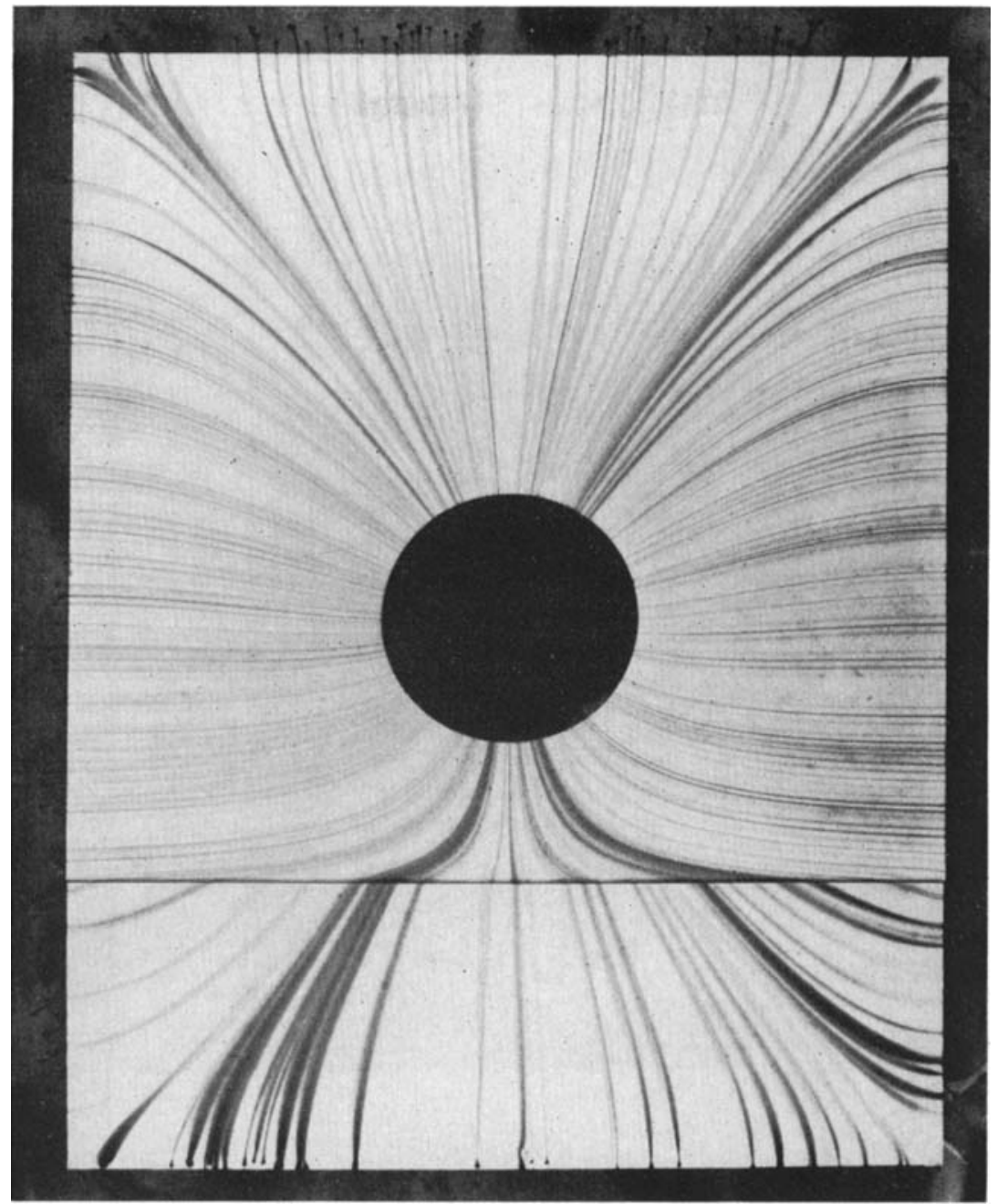

Ficure 3. Fluid mapper simulating a field in two mediums. The two flow spaces are $2: 1$ in thickness, thus representing an $8: 1$ ratio for the dielectric constants, thermal conductivities, ete. Refraction exhibited. A ceiling pattern. The well is a sink.

fluid, however, no trouble is encountered. For longer operations, erosion of the surface due to its going into solution can be almost stopped by first saturating the water with calcium sulfate.

Time and again, during a class demonstration, the writer has cast and finished a simple type of slab and had it in operation within a period of one hour. The presentation of this paper at the conference on which this monograph is based was accompanied by a lecture-demonstration in which a slab was made and operated, from start to finish. This showed the ease and certainty with 


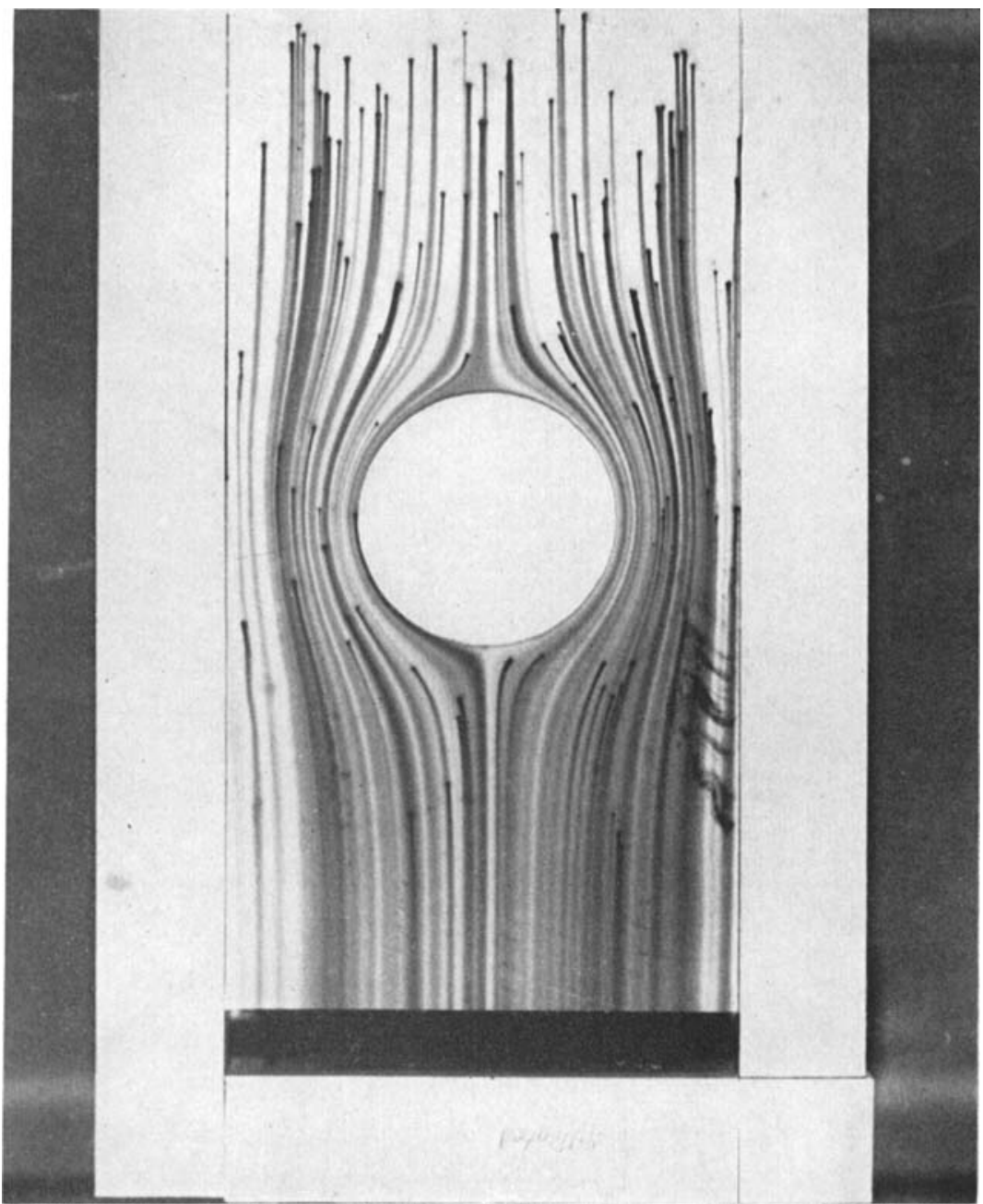

Figore 4. Plaster slab with plaster barriers and plaster island. The crystals rest on the slab, forming a floor pattern.

which plaster techniques can bring the simpler slabs into existence. More intricate slabs will, of course, take more time.

Although the slab of FIGURE 3 shows an intricate pattern, the slab itself was easily made. It was cast in two pieces, the two parts then being joined at the bottom surface by added plaster. The two parts are offset as to upper surfaces, thereby giving the larger area a flow space that is twice that of the smaller area. Owing to a spacing-cubed law ${ }^{3}$ that prevails, we cube the 2:1 ratio and get $8: 1$. This result means we are here simulating a two-medium field and showing the refraction phenomena. For example, if two-dimensional 
Moore: Fluid Mappers for Potential Fields

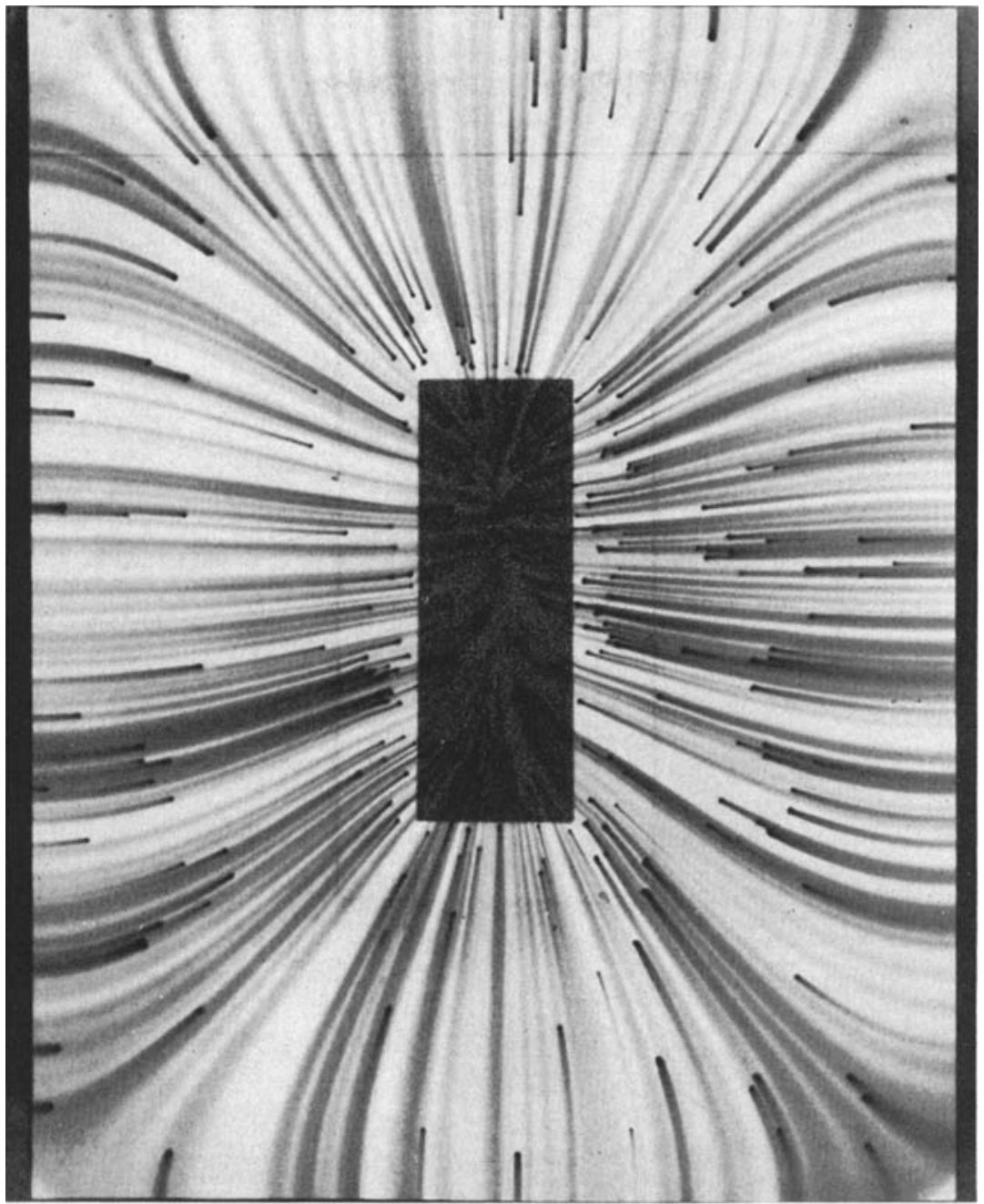

Figure 5. Slab with sandbed as a distributed source. This sandbed is of varying thickness, thereby being a distributed source of nonuniform character. Over the sandbed, Poisson's equation prevails; over the slab, Laplace's equation.

heat conduction is being simulated, then the thermal conductivities represented are in the ratio of $8: 1$.

Flow around an "island" is shown in FIGURE 4. The slab itself was made of four plaster castings, held together with added plaster. The large central casting is depressed to form the flow space. The plate rests upon the narrow white parts, these parts forming the barriers. The round island is a thin casting made between two pieces of plate glass by a "squeeze-out" technique, and later trimmed to roundness. It is as thick as the flow space. It rests upon 


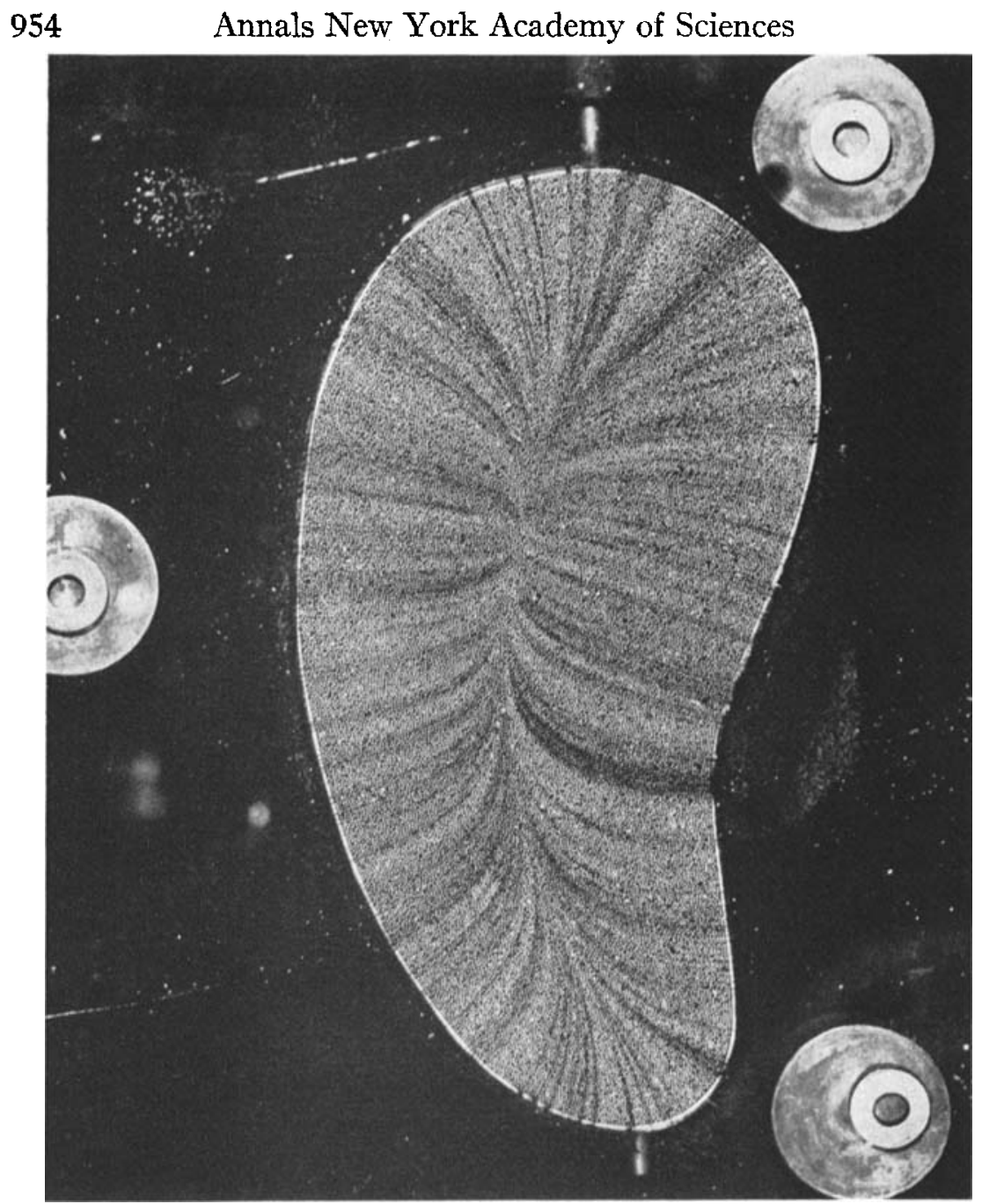

FIGURE 6. Isolated, irregular sandbed acting as a uniform sink. Boundary is an isopressure line. Interpreted as a uniformly distributed heat source, the boundary is an isotherm; the kernel is the hot spot; flow lines delineate heat flow lines.

the slab, and the plate rests upon it as well as on the barriers. With some simple adjustable mold pieces for making rectangular slabs, anyone can soon learn to make a fluid mapper like the one shown in FIGURE 4 and put it into operation.

\section{(4) Flow Spacings}

The thickness of flow space is not a critical matter. Most of the writer's fluid mappers have been operated with a spacing of about 30 to 40 mils. Theoretically, there is no lower limit of flow spacing, provided accuracy is main- 


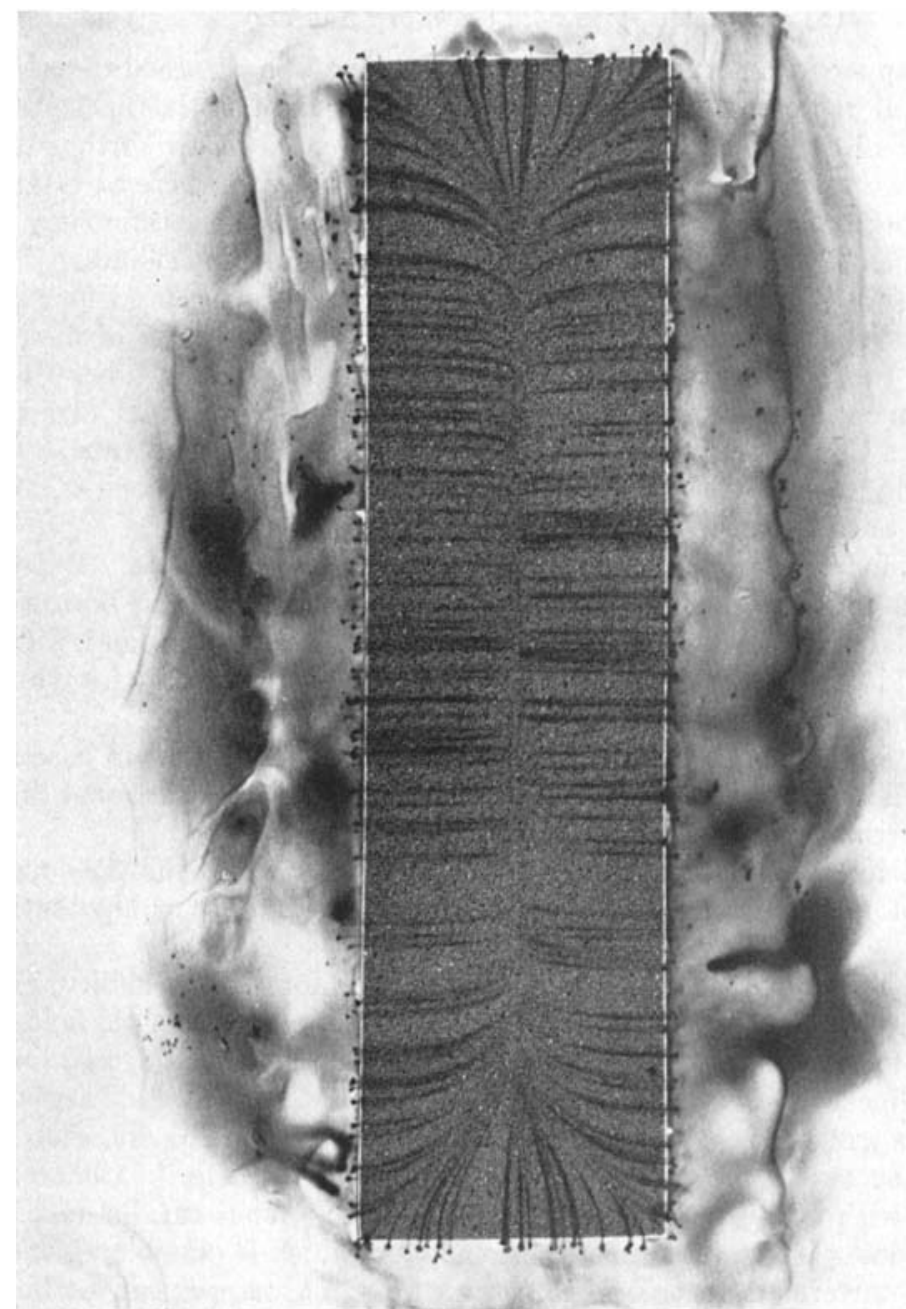

FIGURE 7. Isolated rectangular sandbed acting as a uniform distributed sink. The boundary is an isopressure line.

tained. The upper limit in any particular mapper is reached when flow patterns at different levels no longer agree, and wandering of fluid occurs. No one has been able to work out the upper limit on a theoretical basis; probably, it cannot be done. But experimentation with a specific mapper will determine it, for that mapper, and for the fluid chosen and the velocities used.

Within the wide range of spacings available, one can make a nice demonstration by operating with some value of spacing, and observing the pattern, then doubling the flow space, and operating again. If the upper limit has not been exceeded, the two patterns will be identical. 


\section{(5) Fluid Mappers with Ordinary Sources}

The fluid mappers of FIGUREs 1 to 4 inclusive may all be classified together, in that they all represent "ordinary" two-dimensional potential fields that satisfy Laplace's Equation. Such fields can be represented by two orthogonal sets of lines: two sets of lines, usually curved, that are everywhere at a right angle to each other at their intersections. Using the broadest terminology to cover all such fields, one of the sets of lines is called the equipotential lines. The other set is called flux lines. More specialized names are often used for particular phenomena. If two flux lines are used to delineate a tube of flux, it is an ordinary tube: the flux does not change as we go along the tube. The fluid mappers mentioned above represent such fields, in that if we let two dye lines delineate a flow tube, it will be an ordinary or unaugmented tube. All the fluid entering one end of the tube may be found anywhere along it, and will all depart at the other end.

In FIGURE 2, for example, we see but one set of lines, the flow lines. But we ourselves could carefully draw in the orthogonal system, and then both sets would be available. Which of these would really represent flux, and which potential, would depend on the actual phenomenon being simulated. Three examples will bring out this point (we continue to refer to FIGURE 2):

First, if the fluid mapper is thought of as simulating heat flow in a solid, then the fluid flow lines represent heat flow or flux, and the orthogonal lines represent isothermal (equipotential) lines;

Second, and likewise, if it represents an electrostatic field, the flow lines again represent flux lines, and the orthogonal lines stand for equipotential lines;

But third, if the slab represents iron of high but uniform permeability, and a conductor through the hole has a current which sets up a magnetic field in the iron, then the flow lines represent equipotentials, and the orthogonal lines represent flux lines.

The writer is frequently asked, "Why not build two fluid mappers, each to yield one of the two orthogonal sets of lines of a given case?" Generally speaking, and with rare exceptions, this is impossible. What can be done, if one insists on doing it, is first to make the mapper, which is possible, operate it, and get its pattern on record. From that, a second mapper can be built which will portray some part of (sometimes most of) the orthogonal system. Actually, as far as the writer knows, all field analogs are inherently subject to this same limitation.

Instead of trying to make an analog yield both sets of lines, it is nearly always (and perhaps always) easier to get one set of lines from the analog, and then add the other set by appropriate graphical procedures., ${ }^{3,7}$

\section{(6) The Sandbed}

In FIGURE 2, we have an ordinary well, the edge of which acts as an isopressure line. The well is here acting as a sink. The flow lines leave the outer isopressure boundary orthogonally, and arrive at the well boundary orthogonally. The flow may be reversed, to yield the same pattern. 
If the slab in FIGURE 5 had an ordinary well, with the well acting as a source, then, again, the flow lines would leave its boundary orthogonally. But this is no ordinary well. It is a "sandbed." ${ }^{.4}$ A fine screen has been put across the bottom of this well. A plenum chamber has been built beneath the screen, with the tube connected to it. The plenum chamber is deep enough so that pressure drops in the chamber may be ignored. We have then filled in above the screen with sand or any other suitable material, leveling the sandbed off to the plane of the slab surface. If the "sand" is fine enough, and if the sandbed depth is great enough, the pressure drop up through the sandbed is so great that, essentially, the flow up through the sandbed ignores the fact that slight differences of pressure exist in the flow space above the sandbed. If the sandbed is of uniform thickness, as it is in FIGUREs 6 and 7 , then the fluid is forced to appear or disappear uniformly at the top of the sandbed. In FIGURe 5, the bottom of the well and its screen are sloped, so that the sandbed thickness is three times as great at one end as at the other. The thin part of the bed is nearest to where the kernel, or apparent center of flow, is located.

\section{(7) Sandbed Material}

For his first sandbeds, built in 1943, the writer actually used sand; hence, the name. Sand is quite usable, except for the troublesome fact that it is so light in weight that it is too easily disturbed. After World War II, when other materials became generally available again, metallic "shot" was settled upon as the material of choice. Metallic particles ${ }^{6}$ of nickel, copper, etc., are available. The granules that seem to work best are usually about 14 to 20 mils in diameter.

\section{(8) The Distributed Source}

The sandbed analog is perhaps most easily explained in terms of heat production and conduction. Imagine that, in FIGURE 5, the entire slab represents the rectangular cross section of a long piece of heat-conducting material having uniform thermal conductivity throughout. Let heat be generated or produced in some manner throughout that central part of the solid which is represented by the sandbed. Then the heat would have no choice but to start from that place where some of it is produced, and proceed to flow somewhere. If the entire outer surface of the large piece is maintained as an isotherm, then the heat would have to obey the physical laws of conduction, arrange itself in some pattern of flow, and all arrive orthogonally at the outer boundary.

In the sandbed fluid mapper built to simulate such a case, the fluid is forced to appear in the flow space over the sandbed, and it then has no choice but to flow, by corresponding physical laws; to the open boundary of the slab. By using appropriate sandbed depths here and there, the rate of appearance of fluid over the sandbed is made to copy the distribution of heat production; then the fluid mapper pattern will simulate the actual heat-flow lines.

Distributed-source phenomena occur when heat production is distributed throughout part or all of a volume; when space charges set up electrostatic 
fields; when coil cross sections set up magnetic fields; and in other situations. The field within a distributed source is not the ordinary kind which satisfies Laplace's equation; instead, Poisson's equation prevails. The flux or flow tubes are not "ordinary," with a constancy of flux or flow; instead, we have augmented tubes of flow. The flux or flow increases or decreases, as we follow along a tube.

Until very recently, the world literature offered very few drawings, pictures, or other visual representations of distributed-source field situations. There were three reasons for this scarcity. First, only a very few such fields are simple enough to yield to mathematical attack. Second, these fields are so complex that the attempt to produce even rough sketches of what goes on is often quite beyond the degree of insight available to the human mind. Third, there has been no analog available that could relatively easily portray these situations in visual form. With the advent of the sandbed fluid mapper, this lack is being rectified.

\section{(9) Some Properties of Distributed-Source Fields}

Fields due to distributed sources are so difficult to think about and work with, and adequate visual portrayals have been so scarce, that even those who deal considerably with field theory and field problems are often quite unfamiliar with them. The writer has had an unparalleled opportunity to learn this fact in showing sandbed fluid mapper slides while giving about 56 fluid mapper lecture-demonstrations throughout the country to scientific and engineering groups in colleges, industries, and research units. Four misconceptions or wrong expectations are so commonly entertained that they should be mentioned.

First, those who look at a distributed-source field such as is seen in FIGURE 5 are often prone to think that the kernel is the place where all of the flux or flow originates. This thought is an illusion. Admittedly, it does look that way. Actually, of course, flow tubes starting at the kernel have their flows augmented as they cross the sandbed. In other words, fluid is appearing all over the sandbed, and has to flow from where it does originate. The kernel itself, in a heat-flow case, is the hot spot; or, in a space charge, is the point of high or low potential.

Second, many investigators are surprised to find that the flux or flow is not orthogonal to the boundary of the distributed source. It will be, as in FIGURES 6 and 7, if that boundary is an equipotential line. But in FIGURE 5 this boundary is not an equipotential, and the flow, as shown, crosses it in whatever fashion is called for by the case.

Third, it is commonly expected that, in crossing the source boundary, as in FIGURE 5, refraction will occur. There is no refraction here, provided, as in this case, that the medium is the same throughout. What is present, is a change of curvature. There will be refraction when the distributed source occurs in one medium, and the surrounding field has another medium. For example, if the slab part of FIGURE 5 were to represent the thick wall of a steel tube, and the sandbed part stood for a filling of nuclear reacting material 
producing heat, these two materials having different thermal conductivities, then the heat-flow lines would be refracted at the interface. Correspondingly, the sandbed mapper would then be made up with a sandbed surface above or below that of the slab. It would be a two-space job, representing two media, and the flow lines would, of course, show refraction.

Fourth, if some change is made that is going to shift the position of the kernel, there is a strong tendency on the part of about two out of three observers to predict a movement in the wrong direction. This tendency alone shows how difficult it is to have a true insight into these fields until the mind is trained by seeing a number of cases, and by learning what the factors really are which influence the change to a new conformation.

\section{(10) Isolated Sandbeds}

When a fluid mapper's flow space is confined entirely to the area over a sandbed, the sandbed is isolated-there is no slab surrounding it. The edge or boundary of the sandbed, being open to tray water, is then an isopressure line; the flow lines are orthogonal to the boundary (see FIGUREs 6 and 7 ). The field is all Poissonian, being entirely within the distributed source.

The irregular sandbed of rrGuRE 6 has a definite kernel location, whereas the rectangular sandbed of FIGURE 7 is so elongated that, in effect, the kernal appears to be elongated, becoming a line along the major axis.

FIGURE 6 shows one of the writer's earlier jobs, all-metal except for having a plaster bottom. A great deal of time went into forming a brass strip into a ring, soldering brass rods across it to support a screen, and then fitting a screen onto it to support the sandbed. The sandbed occupies the upper half, and the plenum chamber the lower half.

FIGURE 7 shows a later design, using plaster techniques. Omitting details, be it said that, with plaster rather than with soldering methods, construction is much simpler and easier. The plaster that is seen here, surrounding the sandbed, is wholly constructional. It is depressed considerably below the lip of the sandbed well, permitting the sandbed boundary to operate as an isopressure line. That is, the presence of the plaster, in this mapper, has nothing to do with the pattern over the sandbed.

\section{(11) The Isolated Sandbed as an Analog}

The isolated sandbed is an analog for a number of distributed field phenomena, some of which will be mentioned. Referring to FIGURE 6, if heat-conducting and heat-producing material is uniformly packed into a thin-walled tube, and the tube is maintained as an isotherm, the fluid mapper flow lines duplicate the heat flow lines. If the tube, next, is empty, except for containing a uniform space charge, the flow lines show the electrostatic field conformation. Next, let us remove the filling from the sandbed, put a soap film across the top of the well, and inflate it slightly: the films' contour lines would be orthogonal to the sandbed mapper's flow lines. ${ }^{4}$ The sandbed is accordingly an analog for the soap film, which, in turn, can be used for solving for torsional stresses 
in a shift of like cross section. Thus, the sandbed could be used for solving for torsional stresses.

Consider FIGURE 7 now, and think of the sandbed as representing the cross section of a lamination carrying alternating magnetic flux: the flux lines within the lamination would be orthogonal to the mapper's flow lines. Again, think of this sandbed as being a cross section of a rectangular tube, carrying fluid in streamline flow: the isovelocity lines of the flow would be orthogonal to the sandbed mapper flow lines. ${ }^{3}$

\section{(12) Three-Dimensional Work}

Going beyond the two-dimensional fluid mappers described in this paper, be it said that, sometimes, three-dimensional mappers can be built for fields having axial symmetry. ${ }^{3}$ Such mappers are, of course, more difficult to build. The slab or sandbed surface must be slightly curved, so that the flow-space thickness varies in terms of the radius. There are two types: ${ }^{3}$ In the pro-axis type, the fluid flow is along the axis; in the cross-axis type, it crosses the axis. The flow spacing must vary directly with the cube root of the radius in the pro-axis type and, inversely, with the cube root of the radius in the cross-axis type.

These fluid mapper patterns also are subject to completion procedures, whereby simple graphic and arithmetic techniques bring about the complete solution of the field problem.

One company which has considerable use for three-dimensional fluid mappers has designed and made up metal molds for casting large slabs of both types. The mold surfaces are accurately curved, so as to give the cast slabs their proper curvatures and flow spacings.

\section{(13) Solution of Field Problems}

Whenever a fluid mapper establishes the field conformation, it is possible to photograph or otherwise record the pattern, and then turn it into a complete solution. ${ }^{7}$ This procedure typically calls for selecting particular tubes, mapping the tubes in terms of curvilinear squares, and then going on to do whatever else is necessary to achieve numerical results. Such completion work involves nothing more than the application of graphical and arithmetical routines. A student's understanding of field phenomena is greatly enhanced by doing some of this work, quite irrespective of the training he may already have had in field theory.

Field problems are solved, of course, by a variety of methods. In the relatively small number of cases that are simple enough to yield to mathematics, mathematical attack may be the method of choice. It takes only a little complication, however, such as the imposing of an irregular boundary, or the introduction of a second medium, to put the problem outside the limits of practical mathematical solution.

These more difficult problems must then be handled by the use of graphical field mapping, equivalent networks, or the relaxation method; or by use of the electrolytic tank analog, the conducting paper analog, or the fluid mapper 
analog. The method of choice depends greatly on the actual nature of the problem, on the facilities available, and on the background and training of the individuals bearing the responsibility. There is no one best way to solve all field problems, and there never will be. We need all of the methods we now have, and more besides.

\section{(14) Fluid Mapper Uses}

Many textbooks not primarily devoted to fields nevertheless try to help the student by including some qualitative field sketches. A common example is a sketch of the resultant flux conformation in the case when a current-carrying conductor crosses a magnetic field. The sketch is included to aid the student in seeing why there is a force on the conductor. In many books, this sketch is drawn so that the flux lines bulge away from both sides of the conductor, much as the grain of a pine board would be separated if a nail is driven into the board. Such sketches, being sadly wrong, may do more harm than good. Actually, of course, the field bulges away on one side, but sinks in toward the conductor on the other side. Textbooks commonly in use show many examples of erroneous field sketches. The use of some elementary fluid mappers will, it is hoped, gradually eliminate such atrocities from our literature.

Beyond that, there are large possibilities for enhancing the student's understanding of fields, and his "feel" for them, by illustrating the books with appropriate fluid mapper pattern reproductions. One such book, ${ }^{8}$ Introduction to Electric Fields, by Walter E. Rogers, has already appeared, illustrated in part by such pictures. The writer wishes to take this opportunity to mention the pleasure he derived from working with this author and supplying him with the patterns desired.

Wherever students are learning about potential fields, their insight and understanding of field phenomena may be much enhanced by at least looking at fluid mapper pictures and discussing them. Better yet, some problem solving by way of starting with fluid mapper patterns and completing the analysis to get numerical results can also be included. Still better, a modest fluid mapper laboratory can be established at a very moderate cost, where slab casting and fluid mapper operation can be demonstrated. The colleges are increasingly including such work somewhere in their teaching schedules.

Many teachers are so used to reaching for some instruments on the shelf and quickly setting up a demonstration, with available apparatus, that they may shy away from the business of learning to cast and finish plaster slabs during a demonstration. This procedure need be no deterrent. Slabs can be made up in advance and kept, dry, for indefinite periods. Soaking a slab for a half-hour or less makes it ready for demonstration. Small, thin slabs may need only a ten-minute soak.

Beyond these uses for fluid mappers in teaching and learning, a broad field of application to specific problems may be opened up. Here, of course, fluid mappers are in competition with other methods, and properly so. Fluid mappers do have the great advantage of showing, at once, what the field is like-an advantage offered by no other method. Furthermore. sandbed types 
of fluid mappers may in some cases be able to portray and to lead to the solution of certain distributed-source fields, and do it without competition, no other ready means of solution being available.

The writer developed fluid mappers primarily for their use as analogs for potential fields. However, it was quickly realized by people interested in fluid flow itself, that the techniques devised for making fluid mappers can be directly applied to portray streamline fluid flow in many other cases of interest. Considerable use has been made of fluid mappers for this "direct" or nonanalogous purpose.

\section{References}

1. Heie-Shaw, H. S. \& A. Hay. 1901. Lines of induction in a magnetic field. Trans. Roy. Soc. London. (A) 195: 303-328.

2. Hexe-Shaw, H. S., A. HAy \& P. H. Powell. 1904-05. Hydrodynamical and electromagnetic investigations regarding the magnetic-flux distribution in toothed-core armatures. J. Inst. Elec. Engrs. 34: 21-53.

3. Moore, A. D. 1949. Fields from fluid flow mappers. J. Applied Phys. 20: 790-804.

4. Moore, A. D. 1950. Soap film and sandbed mapper techniques. J. Applied Mechanics. 17: 291-298.

5. Moore, A. D. 1950. Four electromagnetic propositions, with fluid mapper verification. Elec. Eng. 69: 607-610.

6. Moore, A. D. 1950. The further development of fluid mappers. Trans. Am. Inst. Elec. Engrs. 69(2): 1615-1624.

7. Moore, A. D. 1952. Mapping techniques applied to fluid mapper patterns. Trans. Am. Inst. Elec. Engs. 71(1): 1-6.

8. Rogers, W. E. Introduction to Electric Fields. McGraw-Hill. New York, N. Y. 